\title{
Bilayers in amphiphilic mixtures connected by threadlike micelles: a self-consistent field theory study
}

\author{
Martin J. Greenall and Gerhard Gompper \\ Theoretical Soft Matter and Biophysics, Institute for Complex Systems, \\ Forschungszentrum Jülich, 52425 Jülich, Germany*
}

\begin{abstract}
Binary mixtures of amphiphiles in solution can self-assemble into a wide range of structures when the two species individually form aggregates of different curvatures. A specific example of this is seen in solutions of lipid mixtures where the two species form lamellar structures and spherical micelles respectively. Here, vesicles connected by thread-like micelles can form in a narrow concentration range of the sphere-forming lipid. We present a self-consistent field theory (SCFT) study of these structures. Firstly, we show that the addition of sphere-forming lipid to a solution of lamella-former can lower the free energy of cylindrical, thread-like micelles and hence encourage their formation. Next, we demonstrate the coupling between composition and curvature; specifically, that increasing the concentration of sphere-former in a system of two bilayers connected by a thread leads to a transfer of amphiphile to the thread. We further show that the two species are segregated within the structure, with the concentration of sphere-former being significantly higher in the thread. Finally, the addition of larger amounts of sphere-former is found to destabilize the junctions linking the bilayers to the cylindrical micelle, leading to a breakdown of the connected structures. The degree of segregation of the amphiphiles and the amount of sphere-former required to destabilize the junctions is shown to be sensitive to the length of the hydrophilic block of the sphere-forming amphiphiles.
\end{abstract}

*Electronic address: mjgreenall@physics.org 


\section{INTRODUCTION}

When dissolved in solution, amphiphiles such as lipids and block copolymers can selfassemble into a remarkably wide range of different structures $[1,2]$. This phenomenon has been the subject of much recent research $[3,4]$, especially given the potential applications of self-assembled amphiphile aggregates in the encapsulation and delivery of drugs and genetic material $[5,6]$.

In the case of dilute solutions of a single type of simple amphiphile, it is often relatively straightforward to understand, at least on a qualitative level, why a given structure forms in a given system [7]. The major factor in determining the shape of the aggregates is the architecture of the amphiphile; that is, the size of hydrophilic and hydrophobic components. If the hydrophilic component is large compared to the hydrophobic component, then curved aggregates such as spherical or cylindrical micelles form. The hydrophilic component can be intrinsically large (like a bulky head-group) or can appear large due to interaction with the solvent (as for a polymer chain in good solvent). Conversely, if the hydrophobic component is large, lamellar structures such as vesicles are observed.

The picture becomes far more complex when two types of amphiphile that individually form different structures are mixed [1]. In such systems, new structures not seen in monomodal dispersions are observed, such as undulating cylinders [1]. The current interest in such amphiphile blends $[8,9]$ has two major motivations. The first of these is simply that the presence of two amphiphiles increases the number of design parameters available for the control of the self-assembly process. For example, the architectures of both amphiphiles may now be varied, as may the amount of each species. Less obviously, the blending process itself may be manipulated; in particular, the amphiphiles may be mixed together before, during, or after their individual self-assembly [8]. The second factor driving research into blends of amphiphiles is their importance in biological systems. Cells contain mixtures of lipids, which are transported between the various compartments of the cell by mechanisms not yet fully understood [10]. A topic of current debate is the role of membrane curvature in the transport process, and in particular in the sorting of different lipid species within the cell [9]. A model system ideally suited to isolate and investigate the links between curvature and lipid segregation is a large vesicle (of low curvature) attached to a long, thread-like tube (of high curvature) [9, 11]. Examination of the distribution of the different lipid compo- 
nents within this structure then yields information on the connections between membrane curvature, composition, and sorting.

Although this structure is often investigated through the manipulation of individual vesicles using micropipettes [11], recent experiments performed by Zidovska et al. have found similar aggregates consisting of two vesicles connected by thread-like micelles or hollow bilayer-membrane cylinders [12] in bulk solutions of lipid mixtures in a narrow concentration regime. In these experiments, the curvature-stabilizing amphiphiles have an extremely large, branched headgroup, which may account for the fact that threads and tubes form spontaneously here, rather than having to be pulled mechanically from a vesicle (although some mechanical stress produced by flow or by the vesicle formation process may still be present). These authors also explain their results in terms of lipid sorting, and propose a pictorial model of connected vesicles in which the thread contains higher concentrations of curvature-stabilizing amphiphiles than the two flatter bilayers it connects.

We investigate here how much information about connected bilayers can be obtained from a simple mean-field model of two types of amphiphile in solution, without considering details of the shape of the individual lipids or more complex effects such as lipid clustering [9]. In particular, we will investigate the coupling between composition and shape; that is, how addition of a micelle-forming species changes the balance between regions of high and low curvature in the aggregate. The segregation of the different lipid species between these regions will also be studied. Furthermore, we will consider the stability of the junction between the bilayer and a thread, and how this is affected by the addition of micelle-forming amphiphile. Finally, we will consider the effect of varying the architecture of the micelleforming additive on the above phenomena. To study these problems in as simple a form as possible, we will consider two amphiphiles that have no interactions leading to phase separation of the different species. To this end, the amphiphiles will be formed of the same two materials $\mathrm{A}$ and $\mathrm{B}$ but will individually favor different curvatures as a result of the lengths of their hydrophobic and hydrophilic blocks.

The paper is organized as follows. In the following section, we introduce the theoretical technique to be used (self-consistent field theory) and discuss its suitability for the current problem and what information it can yield. We then present and discuss our theoretical results, and give our conclusions in the final section. 


\section{SELF-CONSISTENT FIELD THEORY}

Self-consistent field theory (SCFT) [13] has been used successfully over a number of years to model the equilibrium structures formed in melts and blends of polymers [14-16], and may also be used to investigate metastable structures [17, 18]. Several features of SCFT recommend it as a method to address the current problem of connected bilayers. Firstly, its general advantages are that it requires significantly less computational power than simulation methods such as Monte Carlo, yet often provides comparably accurate predictions of the form of individual aggregates [19-21]. Secondly, as a relatively simple model, with a coarse-grained description of the polymer molecules, it will allow us to capture the basic phenomenology of the system clearly and give insight into how general the phenomena observed are likely to be. In addition, the theory possesses two specific features that are especially advantageous in the current problem. The first of these is that the numerical algorithm that we use to solve the SCFT equations requires an initial guess for the form of the solution. This may be chosen so that solutions of a particular shape, even a rather complex one such as the connected bilayer structure of interest here, may be sought. This is a more direct and straightforward method of systematically studying a given structure than waiting for it to form by chance in a dynamical simulation. The second specific advantage of SCFT is that it makes no initial assumption about the segregation of two structurally different amphiphiles within the self-assembled aggregate, provided the two amphiphiles are formed from the same types of monomer. This will enable us to demonstrate that any such segregation effects arise spontaneously and need not be 'added by hand' to the theory.

To illustrate some of the points made above, and for the sake of concreteness, we now outline briefly the assumptions and mathematical structure of SCFT, and discuss its implementation in the current system. The theory models individual amphiphiles as random walks in space: fine details of their structure and packing are neglected [22]. An ensemble of many such molecules is considered. The interactions between the molecules are modeled on a comparably simple level: by assuming that the blend is incompressible and introducing a contact potential between the molecules [16]. The strength of this potential is specified by the Flory parameter $\chi[23]$.

The first step in finding an approximation method (SCFT) for the above system is to view each molecule as being acted on by a field produced by all other molecules in the system [16]. 
This way of looking at the problem, which as yet involves no approximations, has several advantages when computing numerical solutions. Firstly, it transforms the $N$-body problem of modeling an ensemble of $N$ polymers into $N$ 1-body problems [16]. Since we consider the partition sum over all possible system configurations, all molecules of a given species may be treated as equivalent and we need only solve one 1-body problem for each molecular species in the system. Secondly, this approach allows us to convert the discrete sum over polymer configurations into an integral over smooth functions, which is easier to treat numerically [18]. Finally, the computational difficulty of the problem may be sharply reduced by finding approximate forms of the field variables using a saddle-point approximation [22]. These approximations correspond to neglecting fluctuations in the system.

SCFT can be used to study not only simple homopolymers, but also diblock and more complex copolymers [24] and any given mixture of these [25]. We now discuss the application of SCFT to our system of two amphiphiles in a solvent, which we model by a simple mixture of two types of block copolymer with a homopolymer solvent. Although considerably simpler than the experimental lipid system we wish to consider [12], such models have been successfully used to study biological amphiphilic systems [18], and can isolate individual phenomena more clearly than more complex theories. We take the lamella-forming species of copolymer to have a mean-squared end-to-end distance of $a^{2} N$, where $a$ is the monomer length and $N$ is the degree of polymerization [16]. One half of the monomers in this polymer are hydrophilic (type A) and the other half are hydrophobic (type B). For simplicity, we choose the same value of $a^{2} N$ for the A homopolymer solvent. The curvature-stabilizing species has the same size hydrophobic block as the lamella-former, but necessarily has a longer hydrophilic block. We consider three different mean-square end-to-end distances $\alpha a^{2} N$ for this species, with $\alpha=1.5,2$ and 3 .

In this paper, we keep the amounts of copolymer and homopolymer in the simulation box fixed; that is, we work in the canonical ensemble. This will make it easier for us to access more complex metastable structures, such as the connected bilayer, by a suitable choice of ansatz for the SCFT equations. Such structures are more difficult to stabilize in ensembles where the system is able to relax by varying the amount of the various species, and can require the imposition of detailed constraints on the final shape of the aggregate [18], a step we wish to avoid in our current study of the relationship between composition and curvature.

Applying the procedure described above, we find that the SCFT approximation to the 
free energy of our system has the form

$$
\begin{aligned}
& \frac{F N}{k_{\mathrm{B}} T \rho_{0} V}=\frac{F_{\mathrm{h}} N}{k_{\mathrm{B}} T \rho_{0} V} \\
& -\chi N\left(\phi_{\mathrm{A}}(\mathbf{r})+\phi_{\mathrm{A} 2}(\mathbf{r})+\phi_{\mathrm{S}}(\mathbf{r})-\bar{\phi}_{\mathrm{A}}-\bar{\phi}_{\mathrm{A} 2}-\bar{\phi}_{\mathrm{S}}\right)\left(\phi_{\mathrm{B}}(\mathbf{r})+\phi_{\mathrm{B} 2}(\mathbf{r})-\bar{\phi}_{\mathrm{B}}-\bar{\phi}_{\mathrm{B} 2}\right) \\
& -\left(\bar{\phi}_{\mathrm{A}}+\bar{\phi}_{\mathrm{B}}\right) \ln \left(Q_{\mathrm{AB}} / V\right)-\left[\left(\bar{\phi}_{\mathrm{A} 2}+\bar{\phi}_{\mathrm{B} 2}\right) / \alpha\right] \ln \left(Q_{\mathrm{AB} 2} / V\right)-\bar{\phi}_{\mathrm{S}} \ln \left(Q_{\mathrm{S}} / V\right)
\end{aligned}
$$

where the $\bar{\phi}_{i}$ are the mean volume fractions of the various components. The $\phi_{i}(\mathbf{r})$ are the local volume fractions, with $i=A$ or $A 2$ for the hydrophilic components of species 1 and 2 , $i=B$ or $B 2$ for the hydrophobic components and $i=S$ for the solvent. The strength of the repulsion between the species A (hydrophilic component and solvent) and B (hydrophobic component) is determined by the Flory parameter $\chi . V$ is the total volume, $1 / \rho_{0}$ is the volume of a monomer, and $F_{\mathrm{h}}$ is the SCFT free energy of a homogeneous system containing the same components. The details of the individual polymers enter through the single-chain partition functions $Q_{i}$. As an example, that for the homopolymer is given by [16]

$$
Q_{\mathrm{S}}\left[W_{\mathrm{A}}\right]=\int \mathrm{d} \mathbf{r} q_{\mathrm{S}}(\mathbf{r}, s) q_{\mathrm{S}}^{\dagger}(\mathbf{r}, s)
$$

where the $q$ and $q^{\dagger}$ terms are single chain propagators [16]. The partition functions of the copolymer chains are determined similarly. We now recall that the polymer molecules are modeled as random walks subject to an external field that incorporates their interactions with the rest of the melt. This is reflected in the fact that the propagators satisfy modified diffusion equations. Again considering the case of the homopolymer, we have

$$
\frac{\partial}{\partial s} q_{\mathrm{S}}(\mathbf{r}, s)=\left[\frac{1}{6} a^{2} N \nabla^{2}-W_{\mathrm{A}}(\mathbf{r})\right] q_{\mathrm{S}}(\mathbf{r}, s)
$$

where $s$ is a curve parameter describing the position along the polymer backbone and the initial condition is $q_{\mathrm{S}}(\mathbf{r}, 0)=1$. The copolymer propagators are computed in a similar way, with the copolymer architecture entering into the theory through the fact that the corresponding diffusion equation for the copolymer is solved with the field $W_{i}(\mathbf{r})$ and the prefactor of the $\nabla^{2} q$ term appropriate to each of the two sections of the copolymer [26]. In the case of the longer sphere-forming copolymer, the fields must be multiplied by the ratio $\alpha$ of the two degrees of polymerization [16].

The derivation of the SCFT free energy $F$ also generates a set of simultaneous equations linking the values of the fields and densities at the minimum. The first of these is a simple 
statement of the incompressibility of the system; however, we also find the following linear relation

$$
w_{\mathrm{A}}(\mathbf{r})-w_{\mathrm{B}}(\mathbf{r})=2 \chi N\left[\bar{\phi}_{\mathrm{A}}+\bar{\phi}_{\mathrm{A} 2}+\bar{\phi}_{\mathrm{S}}-\phi_{\mathrm{A}}(\mathbf{r})-\phi_{\mathrm{A} 2}(\mathbf{r})-\phi_{\mathrm{S}}(\mathbf{r})\right]
$$

Furthermore, the homopolymer density is related to the propagators (see Equation 3) according to $[16]$

$$
\phi_{\mathrm{S}}(\mathbf{r})=\frac{V \bar{\phi}_{\mathrm{S}}}{Q_{\mathrm{S}}\left[w_{\mathrm{A}}\right]} \int_{0}^{1} \mathrm{~d} s q_{\mathrm{S}}(\mathbf{r}, s) q_{\mathrm{S}}^{\dagger}(\mathbf{r}, s)
$$

The copolymer densities are calculated similarly, with the integration limits set to give the correct proportions of each species.

In order to calculate the SCFT density profiles for a given set of mean volume fractions, equation 4 must be solved with the densities calculated as in Equation 5 and respecting the incompressibility of the system. To begin, we make a guess for the form of the fields $w_{i}(\mathbf{r})$ with the approximate form of the structure we wish to study and solve the diffusion equations to calculate the propagators and hence the densities corresponding to these fields (see Equations 3 and 5). New values for the fields are now calculated using the new $\phi_{i}(\mathbf{r})$, and the $w_{i}$ are updated accordingly [27]. The procedure is repeated until convergence is achieved.

The diffusion equations are solved using a finite difference method [28] with step size of $0.04 a N^{1 / 2}$. To study the connected vesicle structure, we focus on a region near the junction of the vesicle and the thread-like micelle (Figure 1). The curvature of the vesicle is taken to be sufficiently small that it can be modeled by a flat bilayer. We also assume cylindrical symmetry about the axis of the thread-like micelles, and hence consider an effectively twodimensional problem in a cylindrical calculation box. Reflecting boundary conditions are imposed at the edges of the system, so that we are in effect studying a system of two bilayers facing each other and connected by a thread-like micelle. SCFT is then used to calculate the density profiles of the different species in the system (see inset to Figure 1).

In addition, we perform effectively one-dimensional calculations on spherical micelles, infinite cylinders and infinite bilayers, to complement our calculations on the connected bilayer by demonstrating how the free energy of simple structures with different curvature changes as the volume fraction of micelle-former is varied. At each copolymer composition, the density profile and free energy of the optimum (lowest free energy) sphere, cylinder and bilayer are calculated. The free energies are then plotted to produce a simple phase diagram 
as a function of the volume fraction of micelle former.

The calculation of the optimum aggregates proceeds as follows [29, 30]. Firstly, we calculate the free-energy density of a single spherical, cylindrical or planar aggregate at fixed volume fraction using SCFT. The volume of the simulation box containing the aggregate is then varied until the free-energy density is minimized. This provides a simple model of a larger system (of fixed volume and fixed copolymer volume fraction) containing many aggregates, since such a system can minimize its free energy by varying the number of aggregates and hence the volume occupied by each. Although this approach can be used to perform detailed calculations on micelle formation and shape transitions [29, 30], we use it here to give a demonstration of how copolymer architecture and blending affect the curvature of the self-assembled structures.

\section{RESULTS AND DISCUSSION}

To begin, we calculate the free-energy densities of ideal spheres, infinite cylinders and infinite bilayers using the method of variable subsystem size described above, and determine how these vary as the volume fraction of curvature-stabilizing amphiphile is increased. We fix the overall volume fraction of homopolymer to $10 \%$. The Flory parameter is set to the moderate value of $\chi N=30$ : this is large enough for the copolymers to self-assemble but not so large that all interfaces become sharp. All free energies are plotted with respect to that of the homogeneous solution with the same composition; that is, we plot the quantity $f=F N / k_{\mathrm{B}} T \rho_{0} V-F_{\mathrm{h}} N / k_{\mathrm{B}} T \rho_{0} V$ (see equation 1 ).

As discussed in the preceding section, we use a symmetric lamella-former in all cases, but blend this with different curvature-stabilizing amphipiles. We first consider the strongly sphere-forming amphiphile with mean-square end-to-end distance $R_{0}^{2}=3 a^{2} N$ and fraction of hydrophilic blocks 5/6. Figure 2 shows the free-energy densities of spherical, cylindrical and planar aggregates as a function of $\phi^{\prime} / \phi$, where $\phi^{\prime}$ is the volume fraction of sphere-forming amphiphile and $\phi$ is the total volume fraction of amphiphile (sphere formers plus lamella formers). The free-energy densities $f_{i}$ are quite close in magnitude, and so are plotted normalized with respect to the magnitude of the free-energy density of the cylinder $\left|f_{\mathrm{C}}\right|$ in order to show the shape transitions clearly. The cylinder free-energy density thus appears as a horizontal line at $f=-1$, and is approached from above and below by the sphere 
and lamella free energy densities as the volume fraction of sphere former is increased. At low sphere-former volume fractions, the lamellar structure has the lowest free energy. At around $\phi^{\prime} / \phi=50 \%$, the lamellar and cylindrical free energies cross, and the cylinder has the lowest free energy until $\phi^{\prime} / \phi \approx 70 \%$, when the sphere finally becomes most energetically favorable. There are two important points to note from this calculation in the context of the current problem. Firstly, we show that a blend of sphere-forming and lamella-forming amphiphiles can preferentially form cylindrical structures (such as the threads between vesicles in the experiments of Zidovska et al) [12], even though neither of the amphiphiles forms this structure individually. Secondly, at higher sphere former concentrations, the structures with positive curvature (spheres and cylinders) are shown to have, by some distance, the lowest free energies. This point will be of relevance when discussing the stability of the junctions between bilayers and threads later. We note that the apparent small rise in the sphere-former free energy $f_{\mathrm{S}}$ at low $\phi^{\prime} / \phi$ is an artifact of normalizing the results with respect to $\left|f_{\mathrm{C}}\right|$. In the original units, the difference between $f_{\mathrm{S}}$ and $f_{\mathrm{C}}$ is almost constant for small $\phi^{\prime} / \phi$. However, $\left|f_{\mathrm{S}}\right|$ and $\left|f_{\mathrm{C}}\right|$ decrease steadily as the amount of hydrophobic material in the system decreases. Recalling that $\left|f_{\mathrm{S}}\right|<\left|f_{\mathrm{C}}\right|$, we see that the result of this is that the ratio $\left|f_{\mathrm{S}}\right| /\left|f_{\mathrm{C}}\right|$ decreases slightly, and so $f_{\mathrm{S}} /\left|f_{\mathrm{C}}\right|$ shows the small rise seen in Figure 2.

The shape transitions that occur as increasing concentrations of micelle-former are added to a solution of lamella-forming amphiphile have been studied in detail in the context of lipiddetergent mixtures [31, 32], and the sequence of morphologies calculated above (vesicles, cylindrical micelles, spherical micelles) is indeed observed. These transitions have also been computed using lattice SCFT by Li et al. for a single set of amphiphile parameters [33]. The bilayer-cylinder transition can also be obtained from a much simpler model of membrane curvature [34].

We now consider the effect of adding a curvature-stabilizing amphiphile with a shorter hydrophilic block (but the same size hydrophobic block) to our system of symmetric lamellaformer. The mean-square end-to-end distance of this molecule is $R_{0}^{2}=2 a^{2} N$ and $1 / 4$ of its monomers are hydrophobic. The free-energy density plot is seen in Figure 3. Despite the significant difference in the length of the sphere-forming amphiphile from the case considered above, the transitions from sphere to cylinder and cylinder to bilayer occur at very nearly the same volume fractions as before. The only notable difference between the two figures is that the various free energies are closer together, due to the fact that the two species of 
amphiphile are closer in length than before.

A much more pronounced difference in phenomenology is seen when the hydrophilic block of the micelle-forming amphiphile is shortened still further, so that the mean-squared endto-end distance of this molecule is $R_{0}^{2}=1.5 a^{2} N$ and its fraction of hydrophobic monomers is $1 / 3$. From the free-energy plot in Figure 4, we see that the lamella-cylinder transition is shifted to from $\phi^{\prime} / \phi \approx 50 \%$ to $\phi^{\prime} / \phi \approx 60 \%$, and the cylinder-sphere transition disappears entirely. The implications of this for the stability of connected structures will be discussed later. In addition, and following the trend observed above, the difference between the free energies of the three structures becomes smaller due to the lesser degree of asymmetry between the two amphiphiles.

Having established the basic phenomenology of adding curvature-stabilizing amphiphile to a solution of lamella-former, we now turn our attention to the structure of particular interest here: a system of two flat, parallel bilayers connected by a thread-like cylindrical micelle. As discussed in the methods section, we consider a cylindrical box of copolymer and work in the canonical ensemble. To form the connected bilayer, we initiate the SCFT iteration with a simple ansatz which has the basic form of a connected bilayer, but no detailed information about the curvature of the structure or the distribution of the material between its regions. In the case of a solution of lamella-former with no added sphere-forming species, we find the structure plotted in cylindrical polar coordinates in Figure 5. The plot shows the density of the hydrophilic A blocks, and the cylindrical thread along the $z$-axis appears as a horizontal structure in the centre of the diagram, connecting the two vertical bilayers. Note also the negative curvature of the surface in the regions where the thread joins the bilayers. Junctions of this form have also been seen in lattice SCFT calculations by Jódar-Reyes and Leermakers $[35,36]$ on the closely-related problem of cylindrical micelles bridging two fixed hydrophilic surfaces.

To illustrate the effect of adding sphere-forming amphiphile to the system, we use the following numerical scheme. We fix the number of amphiphilic molecules in the system. Since the two species of amphiphile contain the same number of hydrophobic monomers, this corresponds to fixing the amount of hydrophobic block in the system. However, we gradually replace the symmetric lamella-forming diblocks with sphere-forming copolymers. We first focus on the intermediate-length micelle former with $R_{0}^{2}=2 a^{2} N$. The effect of this on the relative amount of material in the thread and bilayer sections of the connected structure is 
shown in Figure 6, where we show the shape of the structures formed by plotting the total density of hydrophilic A-blocks (lamella former plus sphere former). In Figure 6 (a), only $10 \%$ by volume of all amphiphiles are sphere-forming, and the structure is little different to that formed in a system of pure lamella-formers. In Figure 6 (b), however, we have increased the number of sphere-formers to $25 \%$ of all amphiphiles, and a lengthening of the central thread-like region may be observed. This effect becomes stronger in the plot of Figure 6 (c), where $33 \%$ of the amphiphiles are sphere-forming. We have thus demonstrated how adding sphere-forming species to the system leads to a transfer of material to the more highlycurved cylindrical section, and thus that adding such material may make the formation of thread-like structures more favorable. This is in line with our simpler one-dimensional calculations discussed above, where adding sphere-former was shown to lower the free energy of cylindrical micelles.

However, the existence of connected vesicles such as those seen in the experiments of Zidovska et al. [12]. depends not only on the formation of cylindrical micelles, but also on the stability of the junctions connecting these to the bilayers. In Figure 6 (d), we show the result of replacing $50 \%$ by volume of the lamella-formers with sphere-formers. Here, the cylindrical micelle breaks away from the bilayers that it previously connected, as the regions of negative curvature associated with the junctions become unstable in the presence of large amounts of molecules that strongly favor positive curvature. We note that this solution to the SCFT equations is found when the initial ansatz has the form of a connected structure. Despite having varied the system size and the form of the ansatz, we have been unable to find a connected solution for such high amounts of sphere-former. This is in agreement with the experiments of Zidovska et al. [12], where connected vesicles are found only in a narrow range of sphere-former concentrations. Although adding sphere-former can stabilize thread-like structures, the addition of too high a quantity of such amphiphiles will destabilize the junctions connecting these to vesicles. The competition between these two effects thus restricts the range in which connected vesicle structures may be formed.

To gain further insight into the mechanism behind the growth and eventual splitting off of the thread-like structure, we now consider the distribution of the two amphiphile species within the aggregates. We quantify this using an enhancement factor $\eta(\mathbf{r})$, which we define 


$$
\eta(\mathbf{r})=\frac{\phi_{\mathrm{B} 2}(\mathbf{r})}{\phi_{\mathrm{B}}(\mathbf{r})} \frac{\bar{\phi}_{\mathrm{B}}}{\bar{\phi}_{\mathrm{B} 2}}
$$

This quantity tells us how much the volume fraction of sphere-former is enhanced with respect to that of the lamella-former at a given point in the system. Since $\eta(\mathbf{r})$ is normalized with respect to the total volume fractions of each species, values greater than one correspond to enhancement of the sphere-former concentration and values less than one correspond to depletion.

Figure 7 shows a plot of this quantity within the hydrophobic core region of the connected bilayer formed when $10 \%$ of the amphiphiles are sphere-formers (the system shown in Figure 6 (a)). The segregation of sphere-formers to the central thread can immediately be seen, as can the depletion of this species in the negative curvature regions around the junctions. This result clearly shows the presence of the significant spontaneous sorting of amphiphiles by curvature, even at the current simple level of modeling. Furthermore, it shows that the pictorial model of connected vesicle formation in terms of lipid segregation put forward by Zidovska et al. [12] can be substantiated in explicit calculations.

Further insight into the role of amphiphile segregation in aggregate formation can be gained by plotting the enhancement factor for the higher sphere-former fraction of $33 \%$. This is the highest volume fraction considered before the junctions connecting the bilayers become unstable. In this case, we see in Figure 8 that there is a slightly thinner region in the center of the thread. On either side of this are two slightly darker patches, corresponding to higher concentrations of sphere-former. These features arise from the underlying cylindrical micelle structure of the thread [35]. When the cylindrical micelle breaks away from the bilayers at higher sphere-former concentrations, the clusters of sphere-formers close to the junctions will form the end-caps of the structure shown in Figure 6 (d).

Next, we demonstrate the segregation of the amphiphiles in this split structure. The enhancement factor $\eta(\mathbf{r})$ is plotted in Figure 9. We see immediately that there is a sharp difference in the fraction of sphere-former between the central cylindrical micelle and the flat bilayers. In addition, we note that the range of values taken by $\eta(\mathbf{r})$ has changed: for the two lower sphere-former concentrations shown above, $\eta(\mathbf{r})$ ran from approximately 0.7 to 1.6 ; at $50 \%$ sphere-former, it ranges from around 0.9 to 1.9 . The reason for the increase in the upper limit of the range is the existence of the highly-curved end-caps of the separate cylindrical micelle, which are close to spherical micelles in structure and thus 
strongly favored by the sphere-former. In turn, the disappearance of the regions of negative curvature at the cylinder-bilayer junctions accounts for the rise in the lower limit of $\eta(\mathbf{r})$ to a value closer to one.

Finally, we consider the sensitivity of the amphiphile segregation and junction stability to the structure of the micelle-forming amphiphile. In addition to the sphere-forming additive of mean-square end-to-end distance $R_{0}^{2}=2 a^{2} N$, we have also generated analogous results for the shorter and longer curvature formers introduced earlier, and now present a selection of these.

In Figure 10, we plot cuts through the enhancement factor $\eta(\mathbf{r})$ along the axis of the cylindrical micelle $(r=0)$ for all three micelle-formers $\left(R_{0}^{2}=1.5 a^{2} N, 2 a^{2} N\right.$ and $\left.3 a^{2} N\right)$. In each case, we consider a system where $33 \%$ of the amphiphiles are micelle-formers, as in Figures 6 (c) and 8 above. We immediately see that the segregation of the micelle-forming species to the thread-like micelle increases sharply as its hydrophilic block length is increased. Indeed, the peak value of $\eta(\mathbf{r})$ increases from around 1.35 for the shortest micelle-formers $\left(R_{0}^{2}=1.5 a^{2} N\right)$ to just over 1.9 for the longest $\left(R_{0}^{2}=3 a^{2} N\right)$. We note in particular that changing the mean-square end-to-end distance of the micelle-former from $R_{0}^{2}=2 a^{2} N$ to $R_{0}^{2}=3 a^{2} N$ has a especially strong effect on the segregation. However, the boundaries of the shape transitions between spheres, cylinders and bilayers (Figures 2 and 3) for these two systems are very similar. It therefore appears that the amphiphile structure can have a greater effect on the sorting of amphiphiles than on the shape transitions.

Finally, we also note the effect of amphiphile architecture on the stability of the cylinderbilayer junctions. In the case of the stronger sphere-forming additive with $R_{0}^{2}=3 a^{2} N$, the junctions become unstable when approximately $50 \%$ of amphiphiles are hydrophobic, as in the case discussed above with $R_{0}^{2}=2 a^{2} N$. In contrast, in the case of the shorter amphiphile with $R_{0}^{2}=1.5 a^{2} N$, the connected bilayer remains stable at this composition. The split structure can however be found by increasing the percentage of micelle-former to $67 \%$.

\section{CONCLUSIONS}

Using a coarse-grained mean-field approach (self-consistent field theory) we have modeled several aspects of the formation of vesicles connected by thread-like micelles in amphiphile mixtures. Firstly, we have performed (effectively one-dimensional) calculations on spherical 
micelles, infinite cylinders and infinite bilayers to show that the addition of sphere-forming amphiphile to a solution of lamella-former can lower the free energy of cylindrical structures, thereby encouraging their formation. Next, through more detailed calculations on the connected bilayer structure, we have demonstrated that increasing the concentration of sphere-former leads to a growth of the thread linking the two bilayers. This shows in a direct way how changing the composition of a solution of amphiphiles may change the shape of the aggregates that they form, and, in particular change the relative amounts of regions of different curvature.

Our calculations also yield information on the distrubution of the two species within the structure, and reveal that the concentration of sphere-former is significantly higher in the thread. This is in line with the picture proposed by Zidovska et al. [12] of the connected vesicles observed in their experiments. Furthermore, it shows that the phenomenon of amphiphile sorting by curvature is present even when the molecules are modeled at a very simple level and therefore might expect to be observed in a wide variety of systems. We note that amphiphile sorting is observed in our system even though the two lipids are formed of the same two types of monomer A and B and would not demix in a structure of uniform curvature such as a flat bilayer. This is in contrast to recent experiments by Sorre et al. [9], where proximity to a demixing point is found to be essential for lipid sorting to occur. There are two reason for this. Firstly, we consider amphiphiles that individually form different structures (micelles and bilayers respectively) and so segregate strongly to regions of different curvature within the connected bilayer structure. The amphiphiles used by Sorre et al., however, both form bilayers [37,38] (albeit of different bending rigidities). The extra factor of demixing is thus required for lipid sorting to occur in their system. Secondly, we study bilayers connected by thread-like micelles rather than hollow bilayer tubes. The difference in curvature between the two regions of our structures is thus very large, and a clear segregation of the two species is seen even in the absence of phase separation.

We then investigate the stability of the junctions between the thread and the bilayers, and show that adding larger amounts of sphere-former can cause the connected structures to break down as the regions of negative curvature around the junctions become more unfavorable. Both this effect and the degree of segregation of the amphiphiles are shown to be rather sensitive to the length of the hydrophilic block of the micelle-forming amphiphiles. In particular, the degree of segregation (or efficiency of sorting) is shown to have a particularly 
strong dependence on the hydrophilic block length of the micelle-former.

[1] S. Jain and F. S. Bates, Science 300, 460 (2003).

[2] G. Battaglia and A. J. Ryan, Journal of Physical Chemistry B 110, 10272 (2006).

[3] T. P. Smart, A. J. Ryan, J. R. Howse, and G. Battaglia, Langmuir 26, 7425 (2010).

[4] J. R. Howse, R. A. L. Jones, G. Battaglia, R. E. Ducker, G. J. Leggett, and A. J. Ryan, Nature Materials 8, 507 (2009).

[5] Y. Kim, P. Dalhaimer, D. A. Christian, and D. E. Discher, Nanotechnology 16, S484 (2005).

[6] H. Lomas, I. Canton, S. MacNeil, J. Du, S. P. Armes, A. J. Ryan, A. L. Lewis, and G. Battaglia, Advanced Materials 19, 4238 (2007).

[7] D. J. Kinning, K. I. Winey, and E. L. Thomas, Macromolecules 21, 3502 (1988).

[8] P. Schuetz, M. J. Greenall, J. Bent, S. Furzeland, D. Atkins, M. F. Butler, T. C. B. McLeish, and D. M. A. Buzza, to appear in Soft Matter (2011).

[9] B. Sorre, A. Callan-Jones, J. B. Manneville, P. Nassoy, J. F. Joanny, J. Prost, B. Goud, and P. Bassereau, Proceedings of the National Academy of Sciences of the United States of America 106, 5622 (2009).

[10] K. Akiyoshi, A. Itaya, S. M. Nomura, N. Ono, and K. Yoshikawa, FEBS Letters 534, 33 (2003).

[11] M. Heinrich, A. Tian, C. Esposito, and T. Baumgart, Proceedings of the National Academy of Sciences of the United States of America 107, 7208 (2010).

[12] A. Zidovska, K. K. Ewert, J. Quispe, B. Carragher, C. S. Potter, and C. R. Safinya, Langmuir 25, 2979 (2009).

[13] S. F. Edwards, Proc. Phys. Soc. 85, 613 (1965).

[14] P. Maniadis, T. Lookman, E. M. Kober, and K. O. Rasmussen, Physical Review Letters 99, $048302(2007)$.

[15] F. Drolet and G. H. Fredrickson, Phys. Rev. Lett. 83, 4317 (1999).

[16] M. W. Matsen, Soft Matter (Wiley-VCH, Weinheim, 2006), chap. 2.

[17] D. Duque, J. Chem. Phys. 119, 5701 (2003).

[18] K. Katsov, M. Müller, and M. Schick, Biophysical Journal 87, 3277 (2004).

[19] A. Cavallo, M. Müller, and K. Binder, Macromolecules 39, 9539 (2006). 
[20] C. M. Wijmans and P. Linse, Langmuir 11, 3748 (1995).

[21] F. A. M. Leermakers and J. M. H. M. Scheutjens, Journal of Colloid and Interface Science 136, 231 (1990).

[22] F. Schmid, J. Phys.: Condens. Matter 10, 8105 (1998).

[23] R. A. L. Jones, Soft Condensed Matter (Oxford University Press, Oxford, 2002).

[24] M. Müller and G. Gompper, Phys. Rev. E 66, 041805 (2002).

[25] N. A. Denesyuk and G. Gompper, Macromolecules 39, 5497 (2006).

[26] G. H. Fredrickson, The Equilibrium Theory of Inhomogeneous Polymers (Oxford University Press, Oxford, 2006).

[27] M. W. Matsen, J. Chem. Phys. 121, 1938 (2004).

[28] W. H. Press, B. P. Flannery, S. A. Teukolsky, and W. T. Vetterling, Numerical Recipes in C (Cambridge University Press, Cambridge, 1992), 2nd ed.

[29] M. J. Greenall, D. M. A. Buzza, and T. C. B. McLeish, Macromolecules 42, 5873 (2009).

[30] M. J. Greenall, D. M. A. Buzza, and T. C. B. McLeish, J. Chem. Phys. 131, 034904 (2009).

[31] P. K. Vinson, Y. Talmon, and A. Walter, Biophysical Journal 56, 669 (1989).

[32] J. Oberdisse, O. Regev, and G. Porte, J. Phys. Chem. B 102, 1102 (1998).

[33] F. Li, A. T. M. Marcelis, E. J. R. Sudholter, M. A. C. Stuart, and F. A. M. Leermakers, Soft Matter 5, 4173 (2009).

[34] D. Andelman, M. M. Kozlov, and W. Helfrich, Europhysics Letters 25, 231 (1994).

[35] A. B. Jódar-Reyes and F. A. M. Leermakers, J. Phys. Chem. B 110, 18415 (2006).

[36] A. B. Jódar-Reyes and F. A. M. Leermakers, J. Phys. Chem. B 113, 11186 (2009).

[37] G. G. Shipley, L. S. Avecilla, and D. M. Small, Journal of Lipid Research 15, 124 (1974).

[38] A. S. Ulrich, M. Samia, and A. Watts, Biochimica et Biophysica Acta - Biomembranes 1191, 225 (1994). 
Figures 


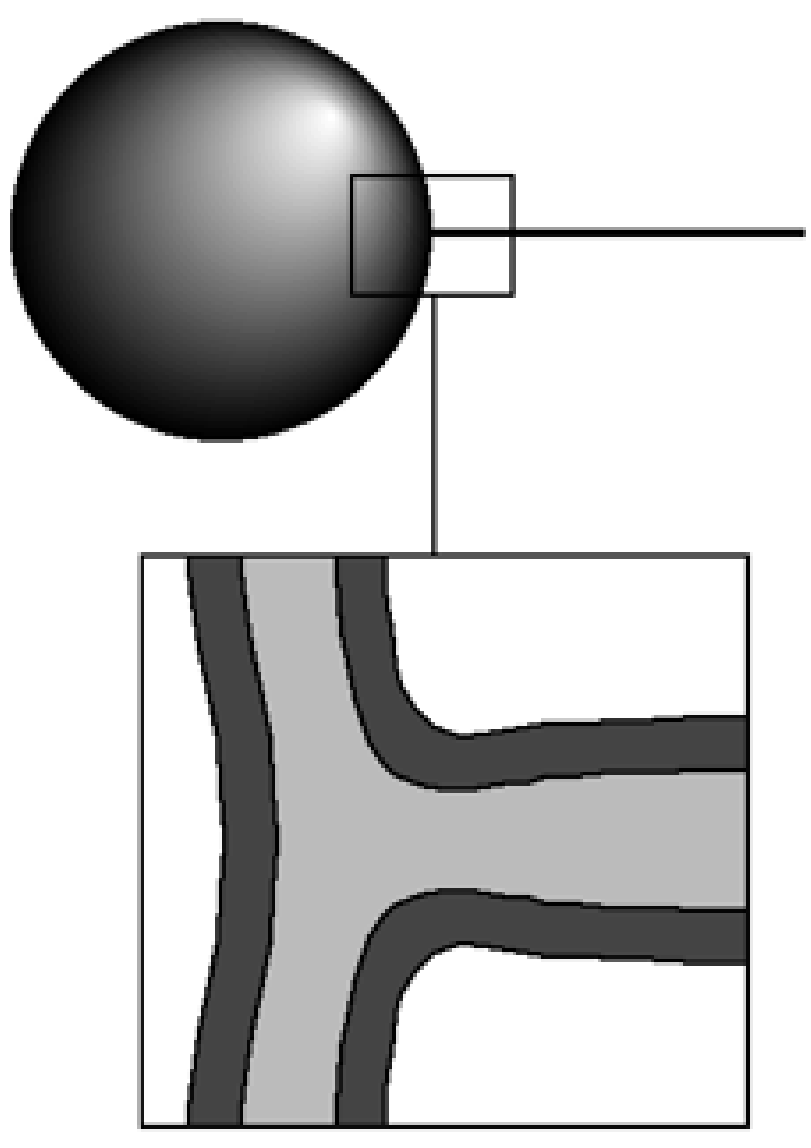

FIG. 1: Schematic of a vesicle connected to a cylindrical micelle. In our calculations, we focus on the junction region sketched in the inset. Here, a vertical cut through the bilayer-cylinder junction is shown. The light region is the hydrophobic core of this structure, and the dark gray region is the hydrophilic corona. In Figures 5 and 6 , we plot the density profile of the hydrophilic corona in order to demonstrate how the structure changes as sphere-forming amphiphile is added to the system. 


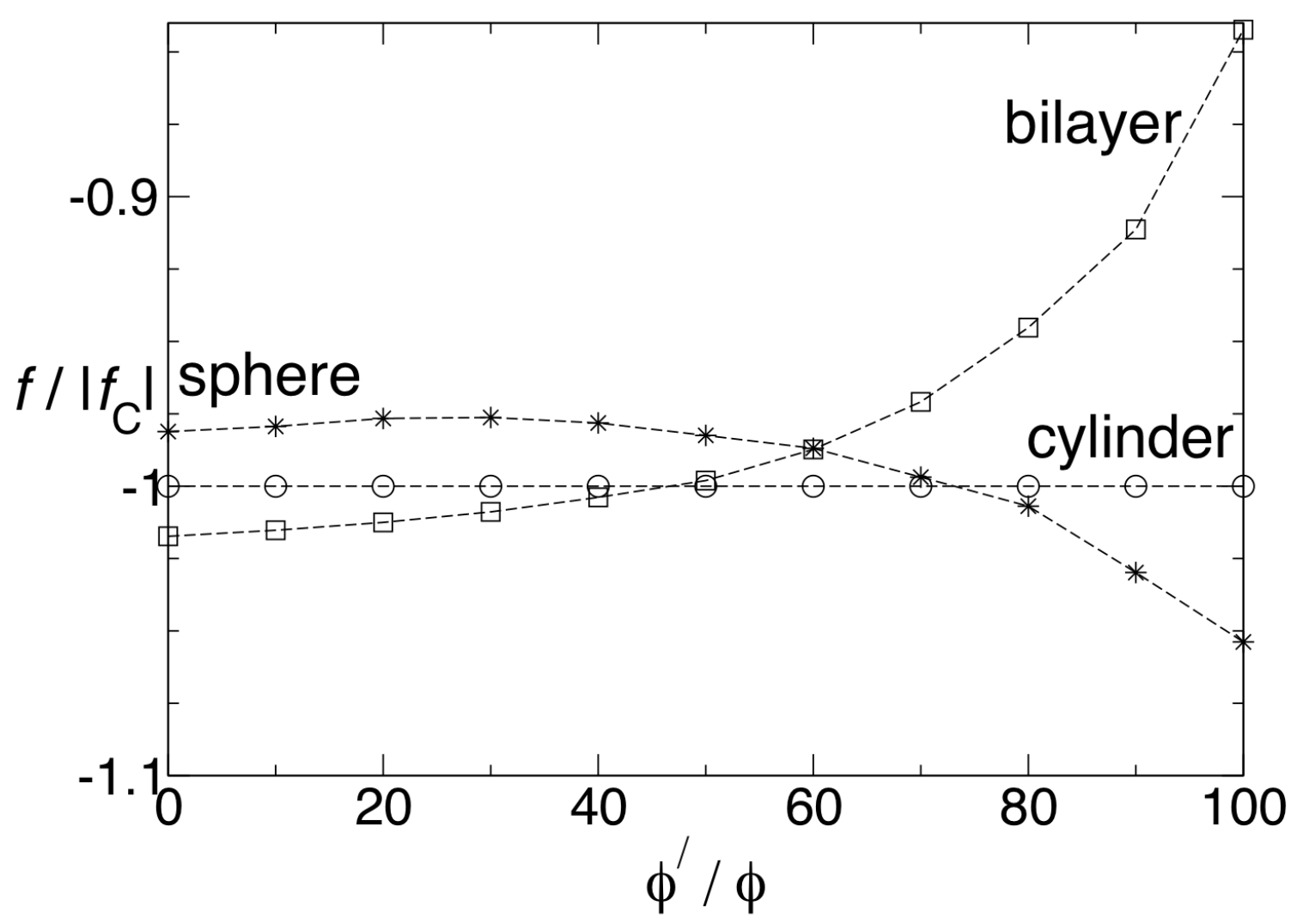

FIG. 2: Free-energy densities of the optimum sphere (stars), cylinder (circles) and bilayer (squares) as the volume fraction $\phi^{\prime}$ of sphere-former is increased at fixed overall volume fraction $\phi=10 \%$. In this case, the micelle-forming species is a strong sphere-former. Its mean-square end-to-end distance is $R_{0}^{2}=3 a^{2} N$, and $1 / 6$ of its monomers are hydrophobic. The Flory parameter takes the moderate value of $\chi N=30$. As $\phi^{\prime}$ is increased, the structure with the lowest free energy changes from the bilayer, then to the cylinder and finally to the sphere. 


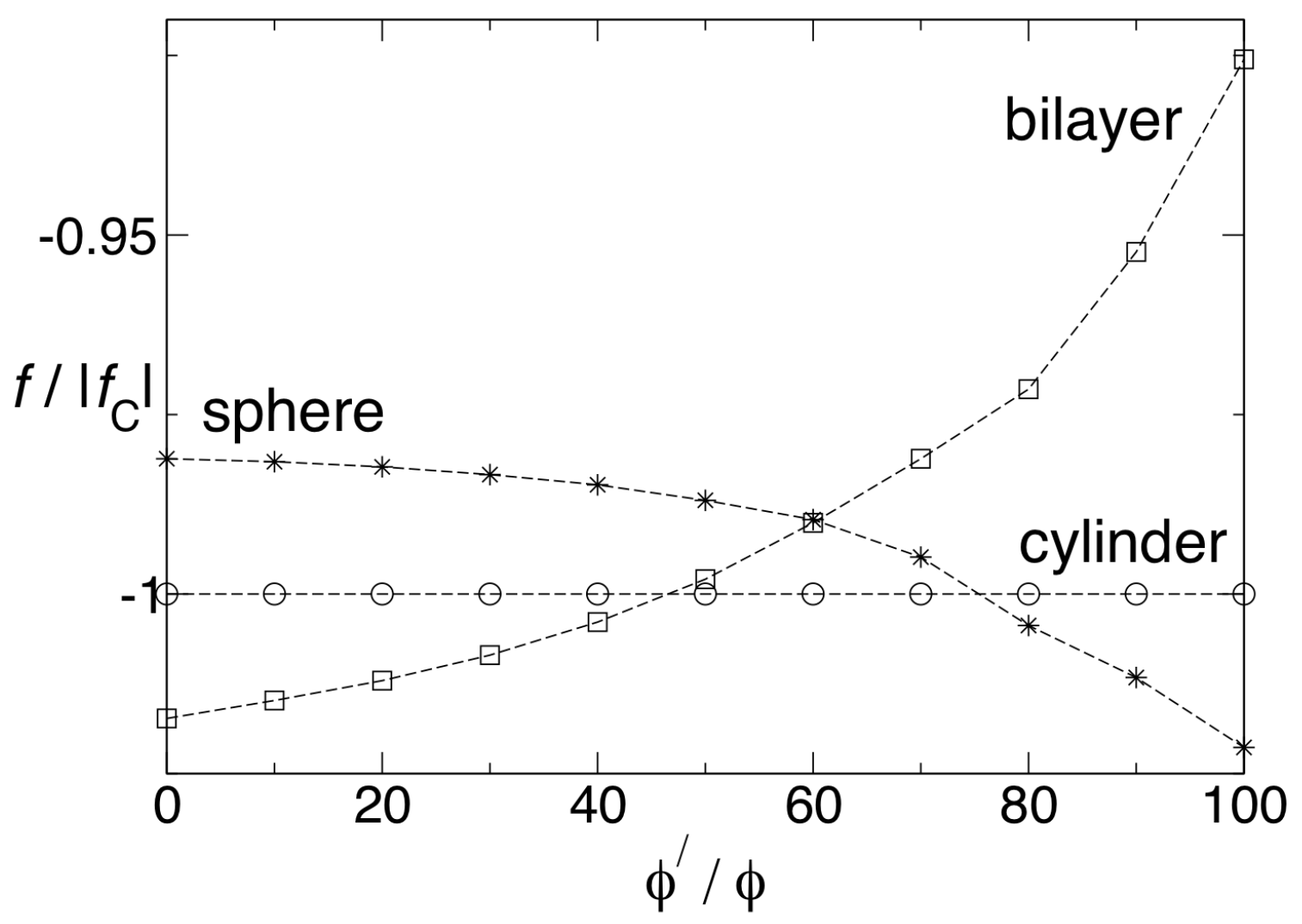

FIG. 3: Free-energy densities of the optimum sphere (stars), cylinder (circles) and bilayer (squares) as the volume fraction $\phi^{\prime}$ of sphere-former is increased at fixed overall volume fraction $\phi=10 \%$. In this case, the micelle-forming species is a sphere-former with $R_{0}^{2}=2 a^{2} N$, and $1 / 4$ of its monomers are hydrophobic. As $\phi^{\prime}$ is increased, the structure with the lowest free energy changes from the bilayer, then to the cylinder and finally to the sphere. 


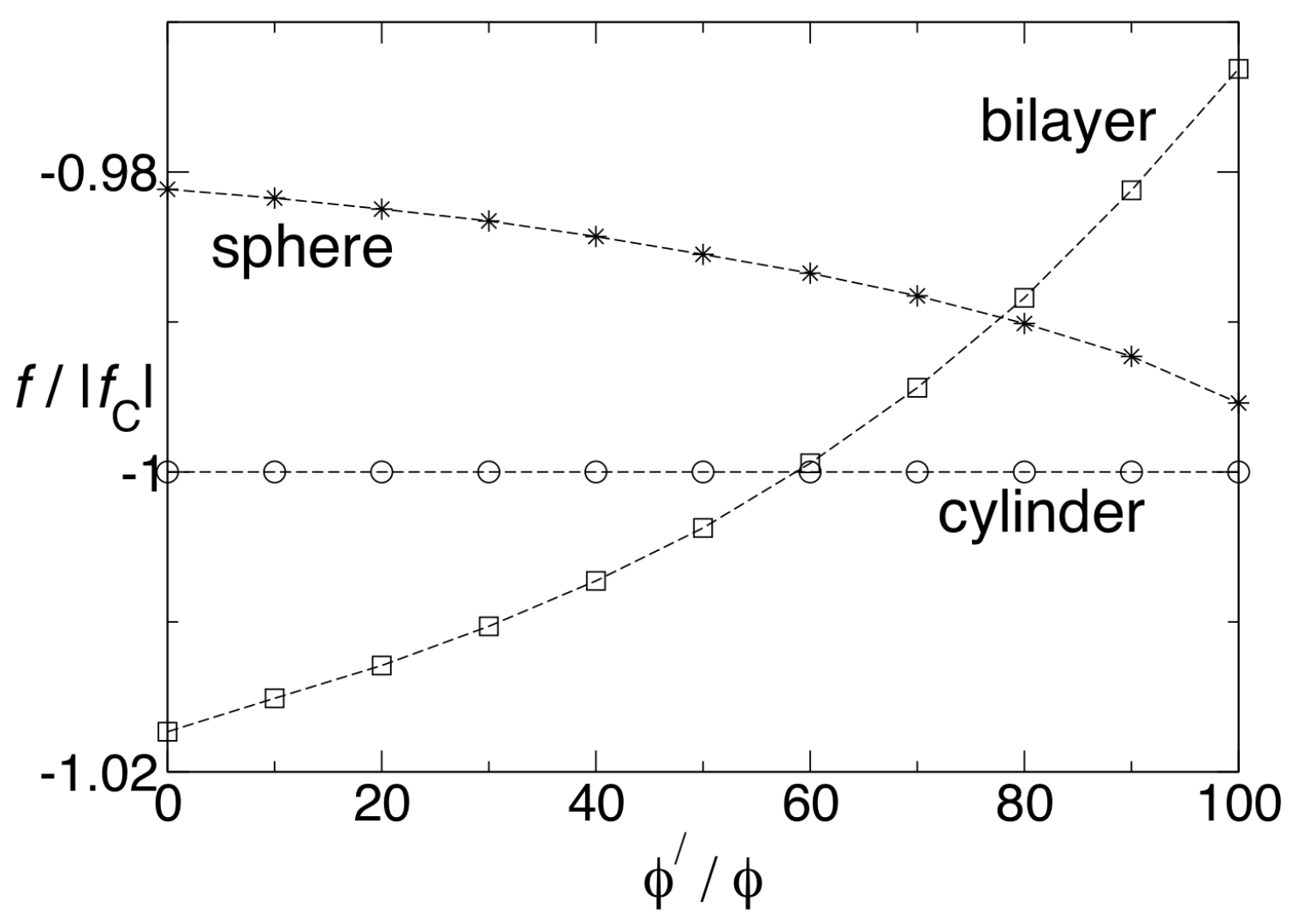

FIG. 4: Free-energy densities of the optimum sphere (stars), cylinder (circles) and bilayer (squares) as the volume fraction $\phi^{\prime}$ of micelle-former is increased at fixed overall volume fraction $\phi=10 \%$. In this case, the micelle-forming species is rather short: its mean-square end-to-end distance is $1.5 a^{2} N$, and $1 / 3$ of its monomers are hydrophobic. As $\phi^{\prime}$ is increased, a transition from bilayers to cylinders is observed, although the cylinder-sphere transition is no longer present. 


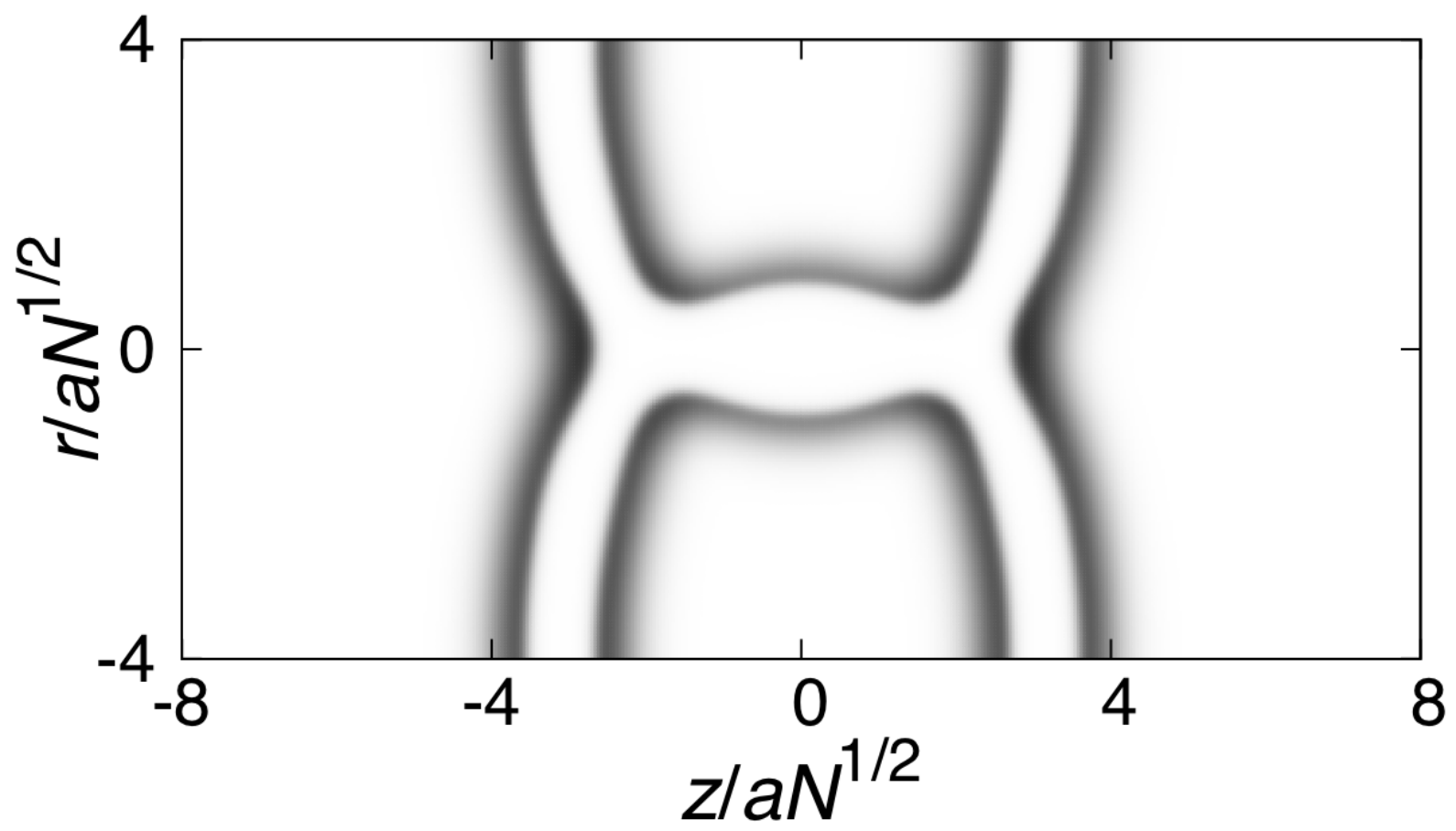

FIG. 5: Density of hydrophilic A-blocks for a connected bilayer structure in a system of symmetric AB amphiphiles dissolved in A homopolymer solvent. Dark regions indicate high hydrophilic block volume fraction. The plot is in cylindrical polar coordinates, so that the connecting thread appears as a horizontal structure along the $z$-axis between the two vertical bilayers. 

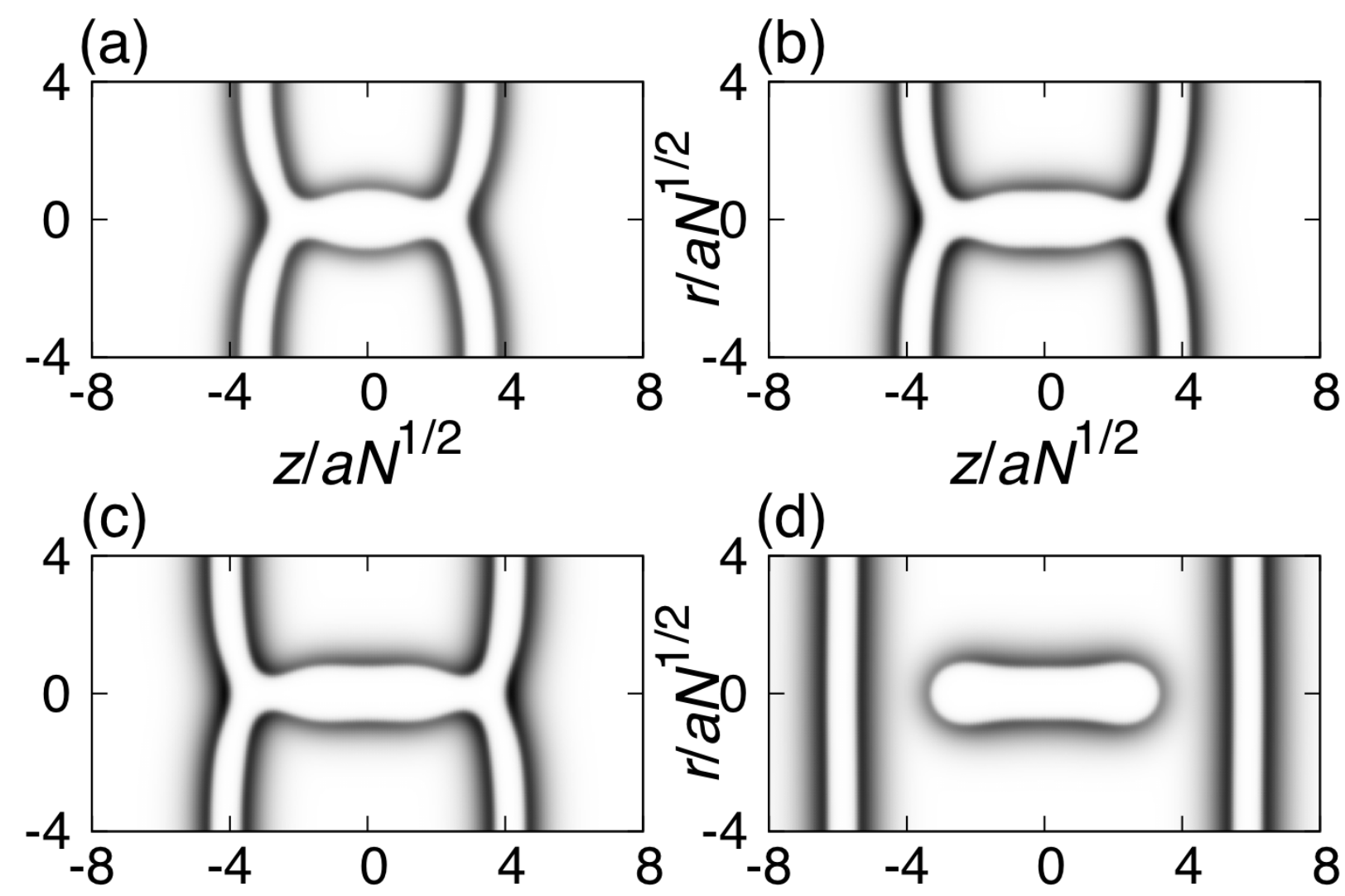

FIG. 6: Total density of hydrophilic A-blocks for connected bilayer structures as lamella-forming polymers are replaced by sphere-formers, keeping the total number of polymers constant. Cylindrical polar coordinates are used, and dark regions indicate high hydrophilic block volume fraction. In (a), $10 \%$ by volume of all amphiphiles are sphere-forming. This is increased to $25 \%$ and then $33 \%$ in (b) and (c), resulting in growth of the thread connecting the two bilayers. In (d), the addition of $50 \%$ sphere-former destabilizes the negative curvature regions around the junctions and leads to a breakdown of the connected structure. 


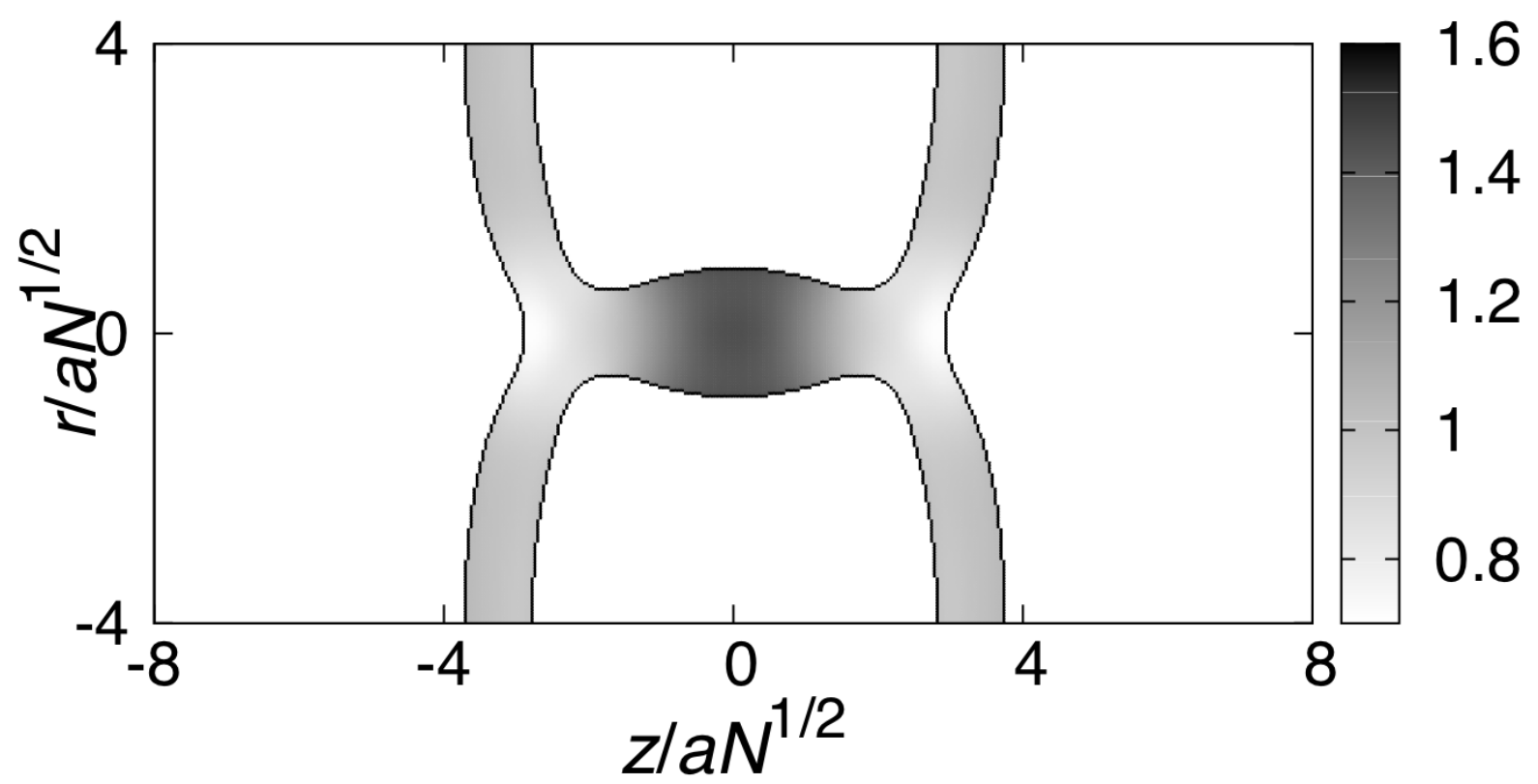

FIG. 7: Enhancement factor $\eta(\mathbf{r})$ in the hydrophobic core region of a connected bilayer formed from $10 \%$ sphere-formers and $90 \%$ lamella-formers. The dark areas $(\eta>1)$ show regions where the local volume fraction of sphere-former is enhanced, and light regions $(\eta<1)$ show regions where it is depleted. The boundary of the core region (defined as the locus of points where the local volume fractions of hydrophilic and hydrophobic blocks are equal) is marked with a black line. 


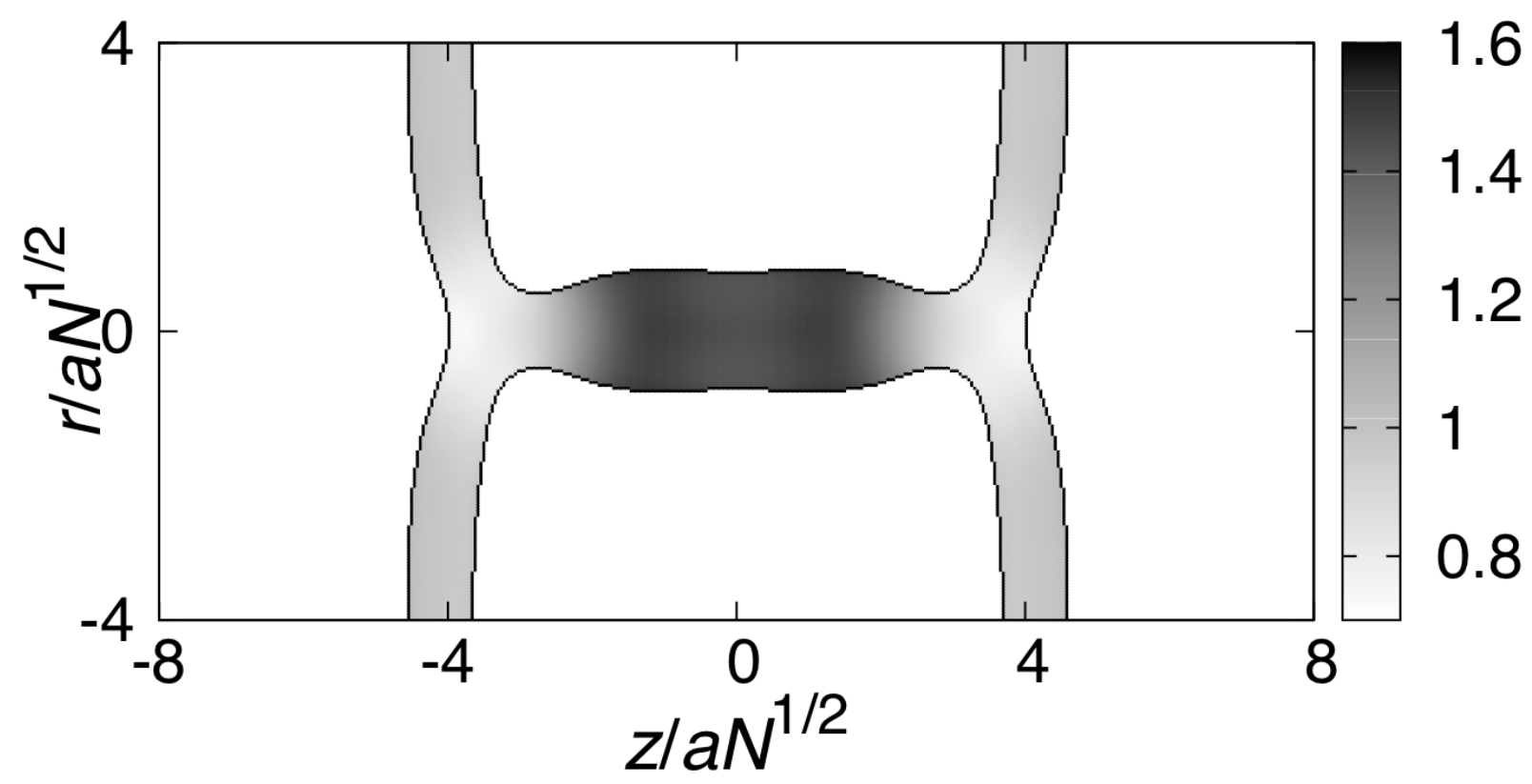

FIG. 8: Enhancement factor $\eta(\mathbf{r})$ in the hydrophobic core region of a connected bilayer formed from $33 \%$ sphere-formers and $67 \%$ lamella-formers. The dark areas $(\eta>1)$ show regions where the local volume fraction of sphere-former is enhanced, and light regions $(\eta<1)$ show regions where it is depleted. A hint of the underlying cylindrical micelle structure can be seen in this result: the sphere-formers have begun to cluster into regions at either end of the thread structure. These clusters will eventually form the end-caps of the separate cylindrical micelle shown in Figure $6(d)$. 


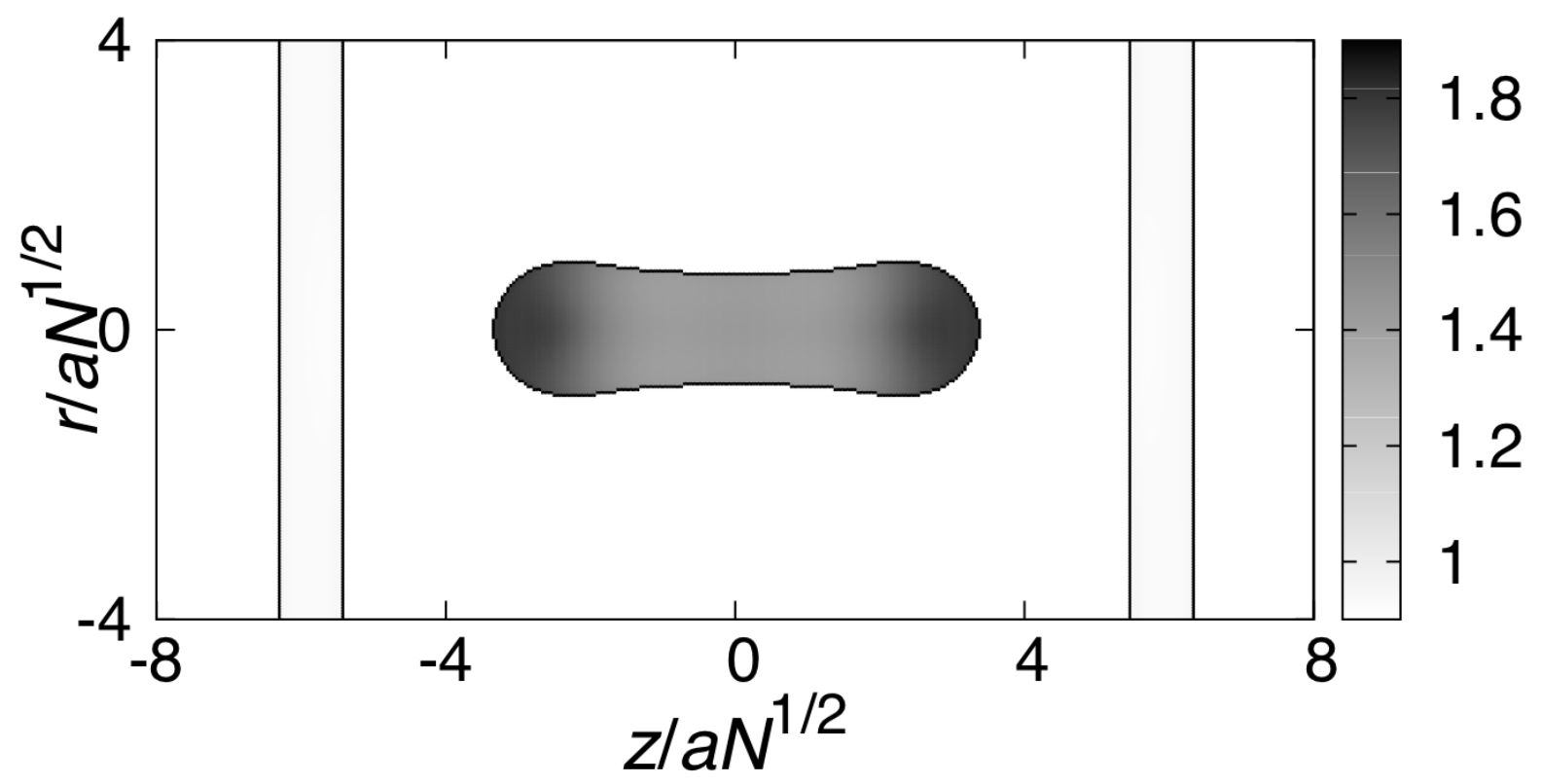

FIG. 9: Enhancement factor $\eta(\mathbf{r})$ in the hydrophobic core region of a split structure (two bilayers and a cylindrical micelle) formed from $50 \%$ sphere-formers and $50 \%$ lamella-formers. The dark areas $(\eta>1)$ show regions where the local volume fraction of sphere-former is enhanced, and light regions $(\eta<1)$ show regions where it is depleted. Note the much higher concentration of sphere-formers in the cylindrical micelle and especially in its end-caps. 


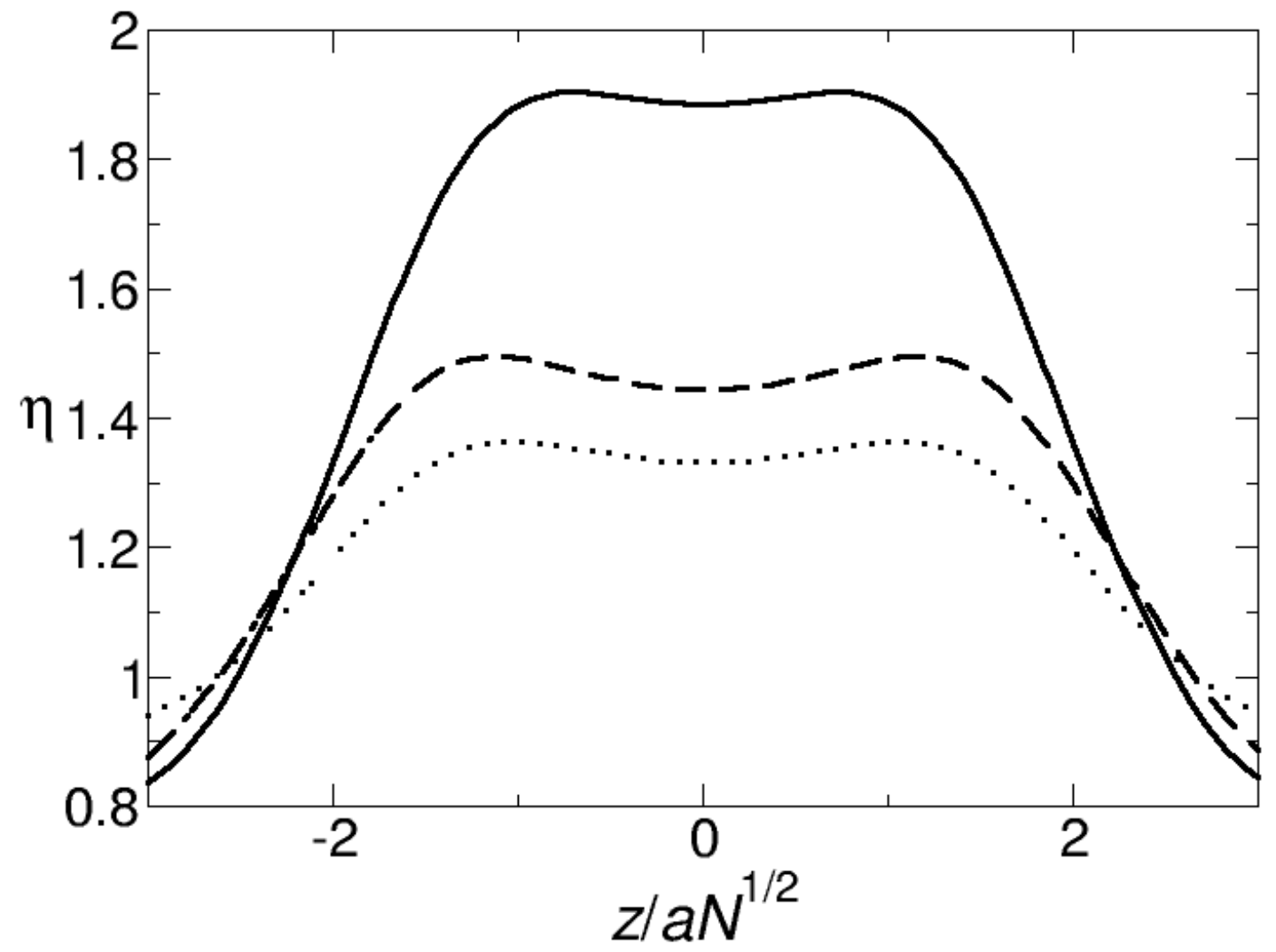

FIG. 10: Cuts through the enhancement factor $\eta(\mathbf{r})$ along the axis of the cylindrical micelle $(r=0)$ for a connected bilayer formed from $33 \%$ micelle-formers and $67 \%$ lamella-formers. The results for the three micelle-formers $R_{0}^{2}=1.5 a^{2} N, R_{0}^{2}=2 a^{2} N$ and $R_{0}^{2}=3 a^{2} N$ are shown with dotted, dashed and full lines respectively. 